\title{
EDITORIAL
}

\section{A geneticist's approach to psychiatric disease ${ }^{1}$}

There is an increasing and welcome hope that genetics may further psychiatric research. If that belief is to be more than mere metaphor, the genetics must be orthodox and not be fancifully moulded to sustain psychiatric ideas, however well founded. Studies have been only modestly successful as compared with the genetics of, say, metabolic disease or blood disorders; and it may not be immediately obvious why. The mutual enrichment of genetics and psychiatry depends largely on the opportunity for clashes between them, much like that between theoretical and empirical science. The clashes should be controlled and sensitive, not antipathetic and destructive. So readers must not be disturbed if much of what follows seems strange, irrelevant, specious, even repugnant; for my goal is not to destroy the relationship, but to map the constraints imposed on it by genetics. My view is not that of a psychiatrist, but of a geneticist; and I shall avoid the tedium of repeatedly saying so. A bill of particulars of the genetics of psychiatry would be large and rather arid. It is more profitable to give a broad, even strategic, perspective in terms of logic and method. It will point to topics of unsuspected richness, only too easily obscured by the details.

\section{THE SETTING}

By genetic standards, most mental disease is both complex and evident.

\section{Complexity}

Mental disease is complex in that it is not immediately and obviously defined by some wholly distinctive character (like achondroplasia), nor by any one chemical criterion (like blood group A). Misdiagnoses are made from time to time, even by experts. So genetic analysis is not straightforward. In this respect, of course, psychiatry is not unique: such complexity occurs in most diseases that are both common and non-trivial: hypertension, diabetes, atherosclerosis. Naive hopes that basically they will prove simple, easily-grasped disturbances continue to remain unfulfilled.

\section{Evidentness}

There are two broad approaches to the genetics of disease: starting from genotype or basic biochemistry (e.g. an aminoaciduria) to finding its psychiatric manifestations; or, starting from an evident state like epilepsy, to seek the underlying mechanisms. Both quests may prove vain. Mental disease is evident: not in being obvious but in the sense that our perceptions of it are rooted in clinical appearances rather than in the familiar paradigms from the exact sciences. Evidentness might be restated in terms of phenotype and genotype. Evident disease may result from a complicated relationship among factors which when analyzed beyond a certain point, disappear without trace. Simple examples are disturbances of symmetry, equality and proportion. That a patient's legs are of unequal length (which may cause scoliosis) will be missed when the two legs are examined by two separate groups of experts not in communication. Only rarely could we infer from data on one leg alone that the patient has scoliosis. Molecular analysis tells us nothing about inequality. More complicated examples arise in the theory of cellular automata (Farmer et al. 1984). The nucleotide is the fundamental, comprehensible, unit of genetics. But the evident phenotype too is important: it is what concerns the patient, the relatives, and current society; and it is on the phenotype that

\footnotetext{
1 Address for correspondence: Professor E. A. Murphy, Division of Medical Genetics, The Joseph Earle Moore Clinic, Johns Hopkins University School of Medicine, Baltimore, Md. 21205, USA.
} 
evolutionary and genetic selection operate. The clinical burden of genetic disease rests on a far coarser scale than molecular biology.

Another radical distinction lies in causation. Genetic aetiology specifies the raw material from which the disease is made. The main tool in its discovery is empirical sampling. In certain cases aetiology is ideally expressed in fundamental chemistry; in other cases in cytogenetic terms or (in classical quantitative genetics) in partition of variances.

Genetic aetiology differs from genetic pathogenesis or medical genetics (Opitz, 1977) in several respects. The forebear of genetic pathogensis is pathology, the study of how aetiological factors are converted into diseases. Aetiology deals, as it were, in ingredients, pathogenesis in recipes. Effective prevention may demand no grasp of pathogenesis. (The streptococcus is a major aetiological factor in rheumatic fever, which can, without more to do, be prevented by antibiotics.) Pathogenesis often involves many steps. Some intermediate state may be called an aetiology e.g. 'The main aetiology of heart attacks is atherosclerosis.' Any such aetiology may be resolved into yet more basic causes. Aetiology is static, pathogenesis dynamic; aetiology essentially formal, pathogenesis essentially empirical; formal aetiology uses statistical models, formal pathogenesis biological ones.

\section{Two patterns of disorder}

As an approach to mental disease, the medical geneticist may provisionally distinguish between two patterns, disruption and aberration.

A Disruption is a disorder in which a system cannot meet the demands made on it. Arterial blockage in a foot, or heart failure, are examples. Mental defect due to injury, infection, or metabolic upset is a disruption. Phenylketonuria is a heritable cause of mental deficiency that bears on the genetics of psychiatry as, indeed, many other metabolic disorders do (Levy, 1973). But the conclusion is privative only. It casts little light on the genesis of normal intelligence: it would not be a rallying point for research on genius. One might as well say, because floods damage carpets, that research on making better carpets should address flood control. Beyond a certain point in the control of either phenylalaninaemia or floods, the disrupting factor ceases to be rate-limiting. We have no reason to believe that carpets may be preserved extraordinarily well merely by being kept extremely dry.

An Aberration is a disorder in which the organism functions coherently but in a way we consider abnormal (Murphy, 1966; Galen \& Gambino, 1975; Feinstein, 1974; Murphy, 1981 a). Thyrotoxicosis and gastric hyperacidity are examples. Commonly aberration is a defect in regulatory processes. Thus, disorders of mood are not obviously a mere dismantling of mental processes: witness the fact that levels of mood may be either greater or less than normal, which hints at a disorder of regulation not scope. It takes discernment to tell where the artistic fantasy of Poe, Blake, or MacDonald shades into the psychotic fantasy in Kafka or (more tantalizingly) into Evelyn Waugh's Ordeal of Gilbert Pinfold.

\section{FEATURES OF ENQUIRY}

In parsing the evidence that bears on the genetics in psychiatry, we shall address three of many issues.

\section{Issue 1: degree of analyticity}

The geneticist grappling with a complex problem has to be concerned with how far his data allow satisfactory inference. Two broad kinds of data are available to medical geneticists; these may be named respectively, catastrophic and manipulative. Key contrasts (Table 1) present a pattern familiar to the pathologist of organic disease. The main virtues of studying the naturally occurring disorder is that it is natural and more or less commonplace, and that, being a real problem, it need seek no special academic or financial warrant: its existence argues its own case. Cancer is common and its clinical management a matter of serious and real concern. We must have clinical and pathological services for confronting it. The main flaw is that the lesions (being spontaneous, undirected, and therefore not logically contained) are coarse-grained. Again, aphasia has mainly 
Table 1. Analytic and synthetic approaches to complex medical problems

\begin{tabular}{|c|c|c|}
\hline & Analytic approach & Synthetic approach \\
\hline Synonym & Catastrophic & Manipulative \\
\hline Setting & The clinic & The laboratory \\
\hline Genetic example & $\begin{array}{l}\text { Impaired spacial sense } \\
\text { in Turner's syndrome }\end{array}$ & $\begin{array}{l}\text { Photic stimulation in } \\
\text { epilepsy }\end{array}$ \\
\hline Methods & Analytical pathology & $\begin{array}{l}\text { Experimental } \\
\text { pathology }\end{array}$ \\
\hline Logical quality & $\begin{array}{l}\text { Variable; often poor; } \\
\text { must be accepted as } \\
\text { it is. }\end{array}$ & $\begin{array}{l}\text { Should be high in } \\
\text { well-designed studies }\end{array}$ \\
\hline Main impediments & $\begin{array}{l}\text { Doubiful logic } \\
\text { Confounding }\end{array}$ & $\begin{array}{l}\text { Ethical constraints } \\
\text { Dubious authenticity } \\
\text { and relevance }\end{array}$ \\
\hline
\end{tabular}

been explored from clinical disorders; and aphasias due to brain tumours or thrombosis lack the resolving power of exquisitely selective, experimental damage.

By contrast, manipulative studies (mostly experiment or provocative tests) are refined, but beset by ethical problems, and open to the charge of artificiality. Also they are elective, often expensive and have to be justified. Finally, for certain problems (in speech, analysis, ideation) no adequate animal model exists, or perhaps can exist. Indeed, such an animal would arguably have the rights of a human being.

\section{Issue 2: complexity}

Two distinct issues arise: intricacy; and polarization.

\section{The level of intricacy}

The problems of studying complex organizations, such as the mind, are widely known. Apart from disruptions, little about the genetics of intelligence has been proved in terms which meet all criticisms levelled against it (Jensen, 1969; Bodmer \& Cavalli-Sforza, 1970; Vogel \& Motulsky, 1979). There are differences in intelligence among species, but in classical theory differences among species are not valid proof of genetic effect: genetics (unlike evolution) deals in traits which are biologically passed from parent to child. It assumes (though in practice not strictly) that the genome has fixed size and structure: both assumptions are expressly abandoned in evolutionary theory. Moreover, comparative biochemistry of the primary structure of the genome shows a similarity (for instance between man and the apes) grossly at odds with the differences in phenotype. How far the differences in intelligence are due to (say) richness of synapsis in the brain, or the higher organization of the DNA (topics under active study) is unknown.

Meanwhile, developing sound intricate tests of intelligence (e.g. psychometrics) is imperilled by their essentially reflexive character: arbiters of intelligence are themselves members of the group being assessed, and they can make no convincing claims to disinterest*. Tests of reproducibility and standardization of the psychometric scales address precision rather than accuracy: they can be validated, but not verified. Of course, this flaw is not confined to psychometry. Nor is it unusually severe there: it merely seems so because the issues involved are sensitive, and intimately human; and psychiatrists have ventilated their concerns about them with rare courage and honesty.

In contrast, the study of aminoacidurias is largely - some would say totally - free of subjectivity in judgment. It is certainly not intricate, and much clean genetic analysis has resulted. Indeed its early success, at the hands of Garrod (1909), laid the foundation for biochemical genetics.

- This partiality offends against a principle encountered in many disparate fields: legal, 'No man shall be judge in his own cause'; logical, notably Russell's paradoxes arising from different levels of language (Russell, 1950); mathematical, Gödel's incompleteness theorem; and of course psychoanalytical notions of rationalization and unconscious motivation. 
At an intermediate level of intricacy lie aneuploidies, which are far from the isolated, primordial, chemical, compounds. Nevertheless, chromosomes are the apparatus of inheritance, and cytologically their transmission can be precisely studied. However, the segregation ratios for chromosomal anomalies are hard to interpret; and in disorders causing sterility, cannot be studied at all. (A strict definition of genetics as the study of transmission of a genome, does not admit the Turner syndrome as genetic. In principle, biochemical genetics would give it warrant.)

Polarization of the enquiry.

The geneticist may aim at 'bottom-up' studies: to build up an understanding of a disease from known elements. Or he may simplify it in 'top down' studies. To these I propose to add a third category that will be less familiar: rearranging the evidence, but at an unchanged level of complexity ('traversal'). The scope is related to the complexity at the starting point; but not wholly determined by it (Table 2).

Table 2. Polarization of the enquiry into complexity of genetic structure

\begin{tabular}{lllll}
\hline & Polarization & Bottom up & Top down & Traverse \\
\hline $\begin{array}{l}\text { Goal } \\
\text { Motive for search } \\
\text { Genetic examples } \\
\text { High complexity }\end{array}$ & $\begin{array}{l}\text { Elaboration } \\
\text { Relevance }\end{array}$ & $\begin{array}{l}\text { Simplification } \\
\text { Identity }\end{array}$ & $\begin{array}{l}\text { Reonentation } \\
\text { Scarlet thread }\end{array}$ \\
Low complexity & $\begin{array}{l}\text { Xependence on } \\
\text { thiamine in } \\
\text { Korsakoff- } \\
\text { syndrome }\end{array}$ & Cretinism & $\begin{array}{c}\text { Quantitative genetics } \\
\text { (e.g. intelligence) } \\
\text { Sickle } \\
\text { haemoglobinopathy }\end{array}$ \\
\hline
\end{tabular}

Bottom-up studies may build up from a simple cause (say an aminoaciduria) to a complex phenotype; or from an aneuploidy to a whole organism. Social psychiatry might address the wider impact of schizophrenia, which is itself a complex phenomenon. Elaboration is a drive towards relevance, towards meaning. A piece of biochemistry, however exquisite, might lead nowhere: we may borrow the term 'orphan' to describe those mutants 'in search of' a clinical (or even evolutionary) significance. Without invoking the longstanding debate over how far polymorphisms are due to drift and to selection (one which in these immensely complicated systems is, I think, quite inadequately, even naively, formulated) we may suppose many mutants are orphans. The 'underlying' defect may be only dimly reflected in the phenotype. More than 30 years ago, Penrose (1951) called attention to this dilution of the genotypic distinctiveness by distance.

Top-down studies driving towards atomizing and simplifying refinement, in ordinary vocabulary called 'reductionism', have had their celebrated successes; they have also had their failures that, far from being deplored, have often been overlooked. There are dangers to the nosological goal of replacing clinical definition by the basic defect as the criterion of disease, on the putative grounds that it is more precise, more scientific. Homocystinuria may cause mental defect; but that does not mean that the blood level of homocystine is a better criterion of mental defect than psychometric assessment, or even than rough clinical judgment. While this mistake has not been made in homocystinuria, the trap has been less successfully avoided elsewhere. Regarding the XYY karyotype as evidence for the diagnosis of aggressiveness has been an unhappy step. It lacks logical justification, even if aggressiveness and this karyotype are indeed associated (a dispute still unresolved).

Top-down followed by bottom-up studies are safer. In many patients sharing a complex phenotype, a common, simple defect is found. Ideally, this defect is used, not to redefine the phenotype, but to define a useful aetiological class, to reduce some of the confusing heterogeneity. It throws light on aetiology rather than on pathogenesis. Its therapeutic value is similar. A diagnosis of homocystinuria suggests the need for a special diet and sometimes supplementary pyridoxine; but at 
the remote level of frank clinical manifestations, homocystine in the urine is not a guide to whether a patient needs remedial education or lens extraction. By contrast, the basic chemical diagnosis has firm value in prognosis and prevention. It allows us to forestall precocious arterial disease and thrombosis. (Arguably all patients with homocystinuria should receive drugs which depress platelet adhesiveness and slow coagulation; and they should remain under ophthalmological supervision.)

The traverse is often overlooked entirely and merits special comment. The bottom-up and top-down approaches at least have a clear goal, even where they should meet with no success. The traverse, a change in format without change in intricacy, is more elusive. Classical quantitative genetics, for instance, hunts for areas of interest by partition of variation, which has by some been taken more narrowly to mean partition of variance and covariance*. The Galton-Fisher theory of analysis of variance (Fisher, 1918), and the use of heritability presuppose the essential unobtrusiveness of every individual aetiological component. If any one major factor stands out, then the axioms are violated; and to reinstate the theory, one must subdivide the data. If sex has any discernible effect, then data pooled by gender violate the assumptions; for Galton-Fisher analysis the data on men and on women should then be logically separated. One cannot at the same time both interpret components of variance and explore the effects of individual constituents.

Two broad methods of examining variation by traversal are familiar to psychometricians: principal components analysis and factor analysis. They aim to extract certain relationships among the data which will recast as systematic effects that which had previously been taken for shapeless random variation. Some 'factors', linear combinations of the data, may lend order, which, it is tacitly supposed, will improve understanding of authentic mechanisms. But even an optimal linear combination of seven variables need not be any easier to grasp biologically than the original seven variables. The factors may, of course, prompt a genetic model; but the statistical analysis is not merely an algorithm that does the geneticists' work for them, even if it gives insight $\dagger$. In this sense the traverse has neither reduced nor elaborated the complexity of the problem, but reorientated it; and the hope is not that it will find the cause, any more than bimodality does, but that it will uncover a scarlet thread that leads to the cause. Trisomy 21 is a feature of Down's syndrome with profound aetiological significance. But its discovery does not mean that the root cause of Down's syndrome is now fully understood. It suggests, perhaps, that some constituent of the chromosome is responsible for the anomalies. But that is not the only, or even the most fruitful, explanation.

Table 3. Focal points of enquiry into the genetics of psychiatric disease

\begin{tabular}{lllll}
\hline & Pathogenesis & Fitness & Aetiology \\
\hline & Analogue & Ingredients & Recipes & Eupepsia \\
Drive & Reductionistic & Integrative & Holistic \\
Discipline & Epidemiology & Pathophysiology & $\begin{array}{c}\text { Clinical and } \\
\text { actuarial arts }\end{array}$ \\
& $\begin{array}{l}\text { Current genetic } \\
\text { commitment }\end{array}$ & Enthusiasm & Almost total neglect & $\begin{array}{c}\text { Neglect or narrow } \\
\text { formalism }\end{array}$ \\
& Formal method & Diverse & Biological modelling & Mainly ecology \\
\hline
\end{tabular}

\section{Issue 3: focus}

We integrate these ideas by identifying the point of our enquiries (Table 3). Aetiology, by nature reductionistic, has simplicity, rigour, and objectivity, the classical virtues of basic science. Advances in genetic aetiology in the last forty years have been spectacular. Issues of fitness and the large-scale holistic behaviour of complicated organisms and societies, have been the mainstay of Darwinism

\footnotetext{
- Variance and variation are not interchangeable terms unless we make stronger appeals, e.g. to the multivariate normal distribution; a broader quantitative genetics would also look at the higher moments and other, perhaps quite different, features of the histogram.

+ A histogram may show bimodality. Yet the 'discoveries' read into the bimodality may mislead. The same applies, more powerfully, to multivariate clusters.
} 
for 130 years; a branch of population genetics was clearly attached to it by Fisher (1958). Yet mathematical genetics becomes steadily estranged from modern high-resolution genetics. Students of genetic pathogenesis are few and their current output exiguous.

\section{THE ONTOLOGICAL AND ANALYTICAL APPROACH}

The concerns of psychiatry show up a nagging strategic weakness at the heart of all rational nosology. In the analysis of disease, what are the rallying points, the criteria and the optimal organization of the raw clinical data? The challenge has been commonly ignored, in the evident belief that, left alone, it will go away. Clinical data pile up, and from time to time they yield facts; but coherent science demands a much firmer skeleton on to which fact and theories may be attached. Psychiatrists confronting these problems explicitly may take comfort from precedent: in the seventeenth century scientists, faced by such a task successfully identified a proper scope and method of physics. Medicine has more slowly, and grudgingly, followed suit.

A few brilliant medical insights have led to revolutions; Pasteur's bacteriology, Bernard-Cannon homeostasis and psychoanalytical theory. But typically they are reached by incisive minds in a carefully thought out context. It is perilous to ignore these contexts, which too often are somewhat dissipated by those exploiting the techniques to which the insights have given birth, to the neglect of the perspective. The discovery of the role of Koch's bacterium distracted attention from an older wisdom about the spread of tuberculosis. Koch saw the difference between necessity and sufficiency; but with the widespread lust for an exact science of a few elemental causes, his admirers for many years paid little heed to his nice distinctions. Reinstatement of this older wisdom was a sound retrenchment from heady absolutism; as a result, tuberculosis was already on the wane before streptomycin, the radical cure, became available.

This craving for oversimplification dies hard. There are still attempts to cast atherosclerosis as an inborn error of cholesterol metabolism, diabetes as a disorder of insulin, hypertension as a defect in handling sodium, osteoporosis as a negative calcium balance. Now any, or all, of these claims may yet prove true. But there are many old familiar arguments against such explanations. On these simplistic terms, why would such diseases be so common? Or chronic? Why do data on the putative causes (cholesterol, glucose, sodium, calcium) in blood or urine not show unambiguous segregation? There are powerful arguments suggesting that aetiology and pathogenesis tend to become complex. For instance, it is easily shown that epistatic (i.e. non-allelic) mechanisms that shelter harmful genes from selection and keep them alive, may be selected for (Trojak \& Murphy, 1983). Multiplicity of genes allows more refined control of a trait and closer clustering near the optimal value than single-locus systems do (Murphy, 1966). There must be many loci controlling fail-safe devices, feedback, repair (Murphy, 1981 b): disturbances in them have as much claim as any to bear on the cause of the disease.

Finding a simple basic cause is, of course, by no means the only way of giving coherence to a mass of data. Thus, a naive view of mood disorders might divide them into those with depression; and those with euphoria. Further experience suggests otherwise; for some patients alternate between the two states, while depression occurs also in organic brain disease, and euphoria in neurosyphilis. So the nosologist changes the criteria and the groupings. A principle of grouping may be useful for many years and then need to be abandoned. If this change is to be a true advance and not merely fashionable reshuffling, there must be some yardstick by which the advance can be judged. Four traditional principles of unity will be cited; but it would be hard to construct a list that is self-evidently complete.

A necessary and sufficient cause may take various forms in genetic psychiatry, including genetic liabilities to infections, such as the sequelae of encephalitis in the cartilage-hair-hypoplasia syndrome; to sensitivity to vitamin intake, such as the impact of thiamine deficiency in Korsakoff's syndrome (Blass \& Gibson, 1977); to intoxications such as occur in Wilson's disease.

$A$ more or less simple pattern of inheritance; one may cite autosomal bipolar mood disorder (Cadoret, 1978); mental retardation in the fragile X syndrome (Turner \& Turner, 1974; Turner \& 
Jacobs, 1983); recent evidence on genetic linkage in Huntington's disease (Gusella et al. 1983). Twin studies are much emphasized by some psychiatrists; but if the pattern of inheritance is at all unclear they are suspect because cultural and environmental effects are hard to sort out from genetic ones, even by exquisite analysis.

Structural concomitants may help; they may be macroscopic, e.g. the form of the skull in Seckel's dwarfism (Seckel, 1960); microscopic, e.g. histological changes in Alzheimer's disease; or cytological, e.g. aneuploidies such as Down's syndrome.

Natural history, i.e. the course that it takes may identify a genetic disorder. Familial Mediterranean fever (Reimann et al. 1954) and acute intermittent prophyria (Waldenstrom \& Haeger-Aronser, 1963), are of special interest to the psychiatrist.

\section{EXPERIMENTAL APPROACHES TO PATHOGENESIS}

My aim in mentioning these approaches further is to show that they are distinct from the issue of complexity: illustrative examples will be taken from all levels.

\section{Top-down studies}

The psychopathological impact of insomnia, an important problem in certain hereditary skeletal disorders, is both complex and well known. One experimental approach to it, sleep deprivation (Webb \& Cartwright, 1978), has explored how dreaming contributes to psychic health, how it is related to REM sleep, etc.

The confusion generated by uninterpretable codes represents a comparable level of complexity but with an elaboration rather than a reduction. Extensions of classical studies by Pavlov have led to the intricate field of frustration theory (Dickenson \& Mackintosh, 1978).

\section{Bottom-up studies}

In some studies (e.g. artificial hyperaminoacidaemia) the manipulation is simple but the consequences complex. Homocystinuria simulated by infusion of homocystine has wide consequences: atherogenesis and shortened platelet survival, both aborted by drugs that depress adhesiveness of platelets (Harker \& Scott, 1977).

Provocative use of DOPA to identify latent Huntington's disease (Klawans \& Goetz, 1980) is a more elaborate bottom-up approach: the chemistry of DOPA is simple, the reasoning underlying the test is subtle, even obscure.

\section{Intermediate levels of complexity}

One may quote the effect of hallucinogens on ideation, and photic stimulation in epilepsy.

\section{SYNTHETIC AND SPECULATIVE APPROACHES}

The methods under this rubric are diverse. Some, e.g. psychoanalytical theory, are hard to put in terms that yield cogent tests*. Twin studies in schizophrenia are only as compelling as studies which make weak surmises about aetiology can be, and none are concerned with pathogenesis.

\section{False models}

I denote by this term those stringent descriptors that rest on mathematical assumptions which are broadly plausible but claim no explicit interpretability for the components. They have more substance than 'the general linear model' of statisticians; but less than (say) the Reed-Frost model of epidemics. An example widely used in recent years is the multiple-hit model. We have used it to study platelet survival (Murphy \& Francis, 1971; Murphy et al. 1973). The broad idea in these models is that a unit, i.e. a single cell or a whole animal, is at continuous risk of outside insults

\footnotetext{
* In psychoanalytical research there has been remarkably little use of genetics: Jung's archetypes would be ripe for exploration if, as seems reasonable, racial memory is genetic.
} 
('hits'). Each hit damages; but, because of repair, fail-safe devices etc, irreparable damage and death occur only after several hits. Models of this kind are called Erlangian. They have a broad, even unassailable, plausibility. Even with no further detail, one can state certain properties about the distribution of the survival time in such a process: it will be unimodal, positively skewed, and, close to Gaussian if the hits are independent, many, and of comparable gravity. These properties explain how a genetic flaw, present from conception, may become manifest late in life e.g. familial polyposis coli (Morales et al. 1984). Alzheimer's disease (Breitner et al. 1986) or familial prolapse of the mitral valve (Strahan et al. 1983). In an Erlangian process if all the hits are of the same size and occur at constant risk $(a)$ and a fixed number $(n)$ is lethal, the gamma distribution results. It has been a favoured model, and appears in more complex form in the bingo model of longevity (Murphy et al. 1981), which supposes that length of life reflects a competition among independent, multiplehit processes.

Despite these qualities and wide use, two features lead me to call the gamma a false, or at best a weak, model.

First, it is too accommodating. It fits well even with Erlangian data from a model that seriously violates the assumptions of the gamma model (Murphy, 1971). Thus it may make it difficult to falsify the model where inept.

Secondly, biologically the model proposes no explicit interpretation of the hits; so it has little heuristic value. Moreover, while the estimate of the mean $(n / a)$, and the variance $\left(n / a^{2}\right)$ are fairly precise, the individual estimates of the component biological features ( $n$, the capacity to recover, and $a$ the hostility of the outside) are highly correlated but individually imprecise. Thus, though a valuable descriptor, the model gives only weak biological information.

\section{A MODEL OF MOOD}

In contrast, I put forward briefly the following instance - whether right or wrong - of an authentic biological model. It is more easily shown false, if false, than the gamma model is; but it will be much richer if it proves true. In a word, it is a dogma, in the (etymologically correct) sense used of the Watson-Crick model of DNA: that is, it is a bold and explicit assertion of fact that will clearly prove true or false.

My model is not cast at the most elemental level, but supposes an authentic phenomenon, mood, that can be manipulated, e.g. by drugs. The course of speculation falls into three stages. Certain clinical facts are identified which merit explanation. The properties of pertinent models of a particular structure are laid out. An adequation of the one to the other is explored in such a way as to yield testable predictions.

\section{A selection of clinical facts about mood}

1. There are cyclical disorders of mood that cause recurrent depression, sometimes with intervening periods of euphoria.

2. The depression is sometimes clearly precipitated by environmental factors, which used to be called reactive or 'exogenous' depression, but is often apparently unprovoked 'endogenous' depression.

3. At least some forms of this cyclical disorder of mood seem to be inherited, perhaps as an almost pure Mendelian autosomal dominant (Cadoret, 1978).

4. Some cyclical variation in mood occurs in people who, by any reasonable standard, are mentally normal; but the normal swings are manageably small. Also, when subjects with the bipolar mood disorder (Folstein et al. 1982), or even normal subjects (Judd et al. 1977), are treated with lithium, the swing in their mood is dampened.

\section{Properties of Homeostatic Systems (Murphy \& Pyeritz, 1986)}

We are concerned here with those physiological processes that attempt to maintain a bodily trait at a particular level termed the homing value by counteracting perturbations of it by outside factors. 
In general, the displacement will lead to a correction that has an opposite sign. The response may be proportional to the size of displacement raised to some power (the order of the process), the constant of proportionality being termed the correction constant.

1. Homeostasis is widespread in higher organisms, and presumably has important selective advantages (Murphy, $1981 b$ ).

2. Homeostasis cannot at once correct displacements; for relaying messages about a displacement and mobilizing responses, e.g. secretion of more hormone, will involve a certain lag-time. The lag has a lower limit, but it may be indefinitely prolonged. Where the correcting force continues to be activated so long as discrepancy persists it will remain one lag-time out of date throughout.

3. The vigour of the homeostatic response must be kept within limits. If too feeble, it serves little purpose. Because of the lag, which acts somewhat like inertia, the correction coefficient if too great will lead to disabling overcorrection.

4. If the response is proportional to the displacement raised to some fractional power, any displacement, however small, sets up a permanent, stable oscillation. Its periodic time and amplitude depend only on the length of the lag time and the vigour of the response. The larger either may be, the bigger the oscillation. The size of the initial displacement has no lasting impact on amplitude. (This oscillation is produced so simply that one supposes it may have been exploited by evolution in any naturally cyclical function.)

\section{Testable surmise (the biological model)}

1. Mood is controlled by a homeostatic process of fractional order, and hence cyclical. This cyclical pattern may have certain selective advantages. It is a device for buffering certain shocks (e.g. grief). It provides a means of regulating circadian or other cyclical functions (heart beat, sleep rhythms, menstruation, annual variation in body fat, etc).

2. Much fluctuation in mood is possible (Fig. 1) within safe limits. However, if the homing value is low, big swings downward may drift into the range of frank depression. When the contrary happens, pure recurrent euphoria is to be expected.

3. Exaggerated responses might be due to increase either in the correction coefficient or the lag time, or both. Since any displacement, however small, may act as the trigger, the lag time would

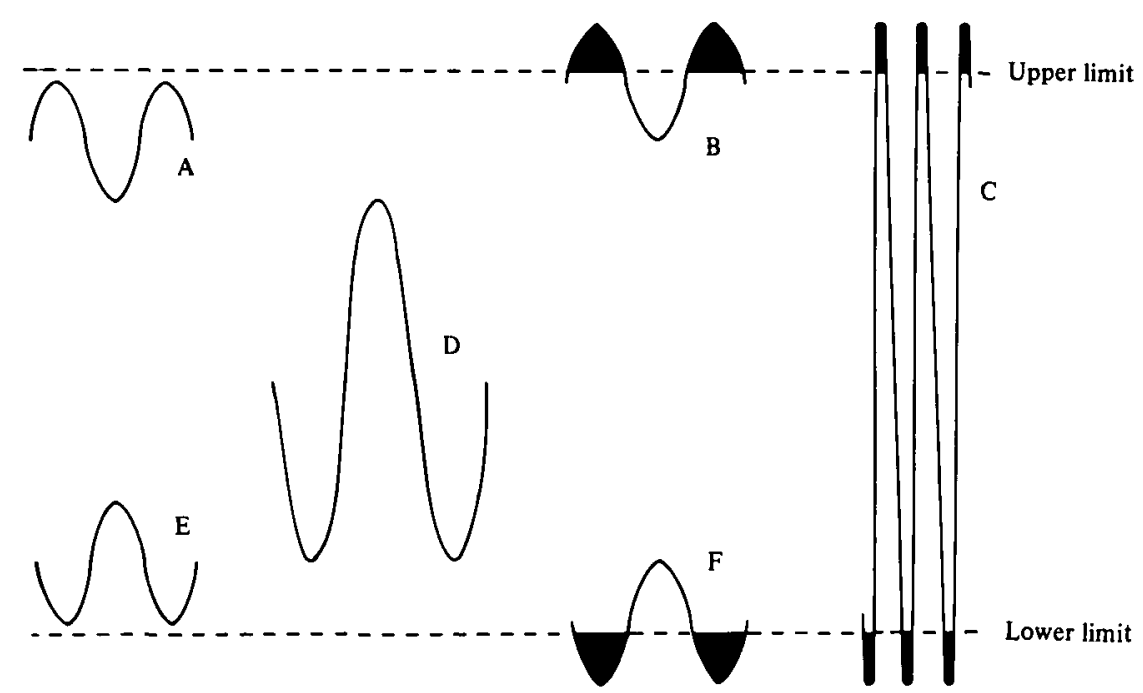

FIG. 1. Phenotypes associated with various combinations of means and amplitudes of perpetually oscillating homeostatic processes of fractional order. Areas in black indicate the fraction of the cycle that leads to mania (above upper limit) or depression (below the lower limit of normal). A, high homing value, low amplitude; B, same amplitude but slightly higher homing value, cyclical mania; $C$, homing value near average, but very large amplitude, alternating mania and depression; $D$, homing value near average with fairly large amplitude; E, low homing value, small amplitude; F, slightly lower homing value and small amplitude, recurrent depression. 
not in general be measurable directly; however it could be estimated by detailed analysis of the parameters of the mood curve.

4. If this model is indeed valid, it is rational to seek for genetic factors in the lag time and the correction coefficient of the response (which would lie nearer to the site of action of the putative gene) rather than in the mood level which is a high level and remote manifestation.

5. Moreover, the parameters would not be obscured by the homing value for the mood; hence one would not have recourse to formless, unilluminating, and non-falsifiable words such as 'incomplete penetrance' to explain why genetic transmission in pedigrees is not perfectly regular.

6. The extent of the mood swing and the impact on it of lithium could be analysed. Perhaps antidepressant drugs act on the mood, lithium on the swing (Folstein et al. 1982; Judd et al. 1977). Detailed studies would make for a more rational use of these drugs. Treatment could act by changing the correction constant or the lag time; or by acting on the mood directly, and in effect producing a perturbation offsetting the original perturbation.

7. The most effective use of drugs would involve an unfamiliar strategy. When euphoria is at its height, it will initiate the strongest trend towards the opposite state, depression. A rational management would be to treat with drugs that support euphoria, timing them so as to anticipate this overshoot. If the interval required for the drug to work is long, it may then be necessary to take the frankly counterintuitive step of treating a patient already euphoric with euphoriant drugs. The optimal scheme would depend on the details of the pharmacology of the drug, which fall within the domain of empirical data. These predictions, unless verified, would not of course form any rational basis for management of affected patients.

\section{SUMMARY}

The pattern of the genetics of psychiatric disease is various, in detail and in kind; one cannot always expect to find simple, trustworthy explanations of what are complex relationships. There will be some diseases, mostly of the disruptive type, that will prove to be Mendelian defects; but it would be idle to expect that of most. Many disorders will be elucidated only when the nub of the problem is better defined. Mindless application of standard genetic techniques devised for quite different purposes is no substitute for articulate speculation built on sound fact and cogent testing. In this respect genetic evidence is no different from that of physiology, pathology and pharmacology, which are perhaps less unfamiliar to readers than genetics. Finally, no answer has been found unless it deals in those terms in which the question first arose. If the topic of the genetic analysis is some psychosis, the final predicate must be a statement about the psychosis, not about some arbitrary abstraction from it.

EDMOND A. MURPHY

The preparation of this paper was supported by grant number GM 34152, from the National Institutes of Health.

\section{REFERENCES}

Blass, J. P. \& Gibson, G. E. (1977). Abnormality of a thiaminerequirng enzyme in patients with Wernicke-Korsakoff syndrome. New England Journal of Medicine 297, 1367-1370.

Bodmer, W. F. \& Cavalli-Sforza, L. L. (1970). Intelligence and race. Scientific American 223, 419-429.

Breitner, J. C. S., Folstein, M. F. \& Murphy, E. A. (1986). Familial aggregation in Alzheimer dementia I. A model for the agedependent expression of the autosomal dominant gene. Journal of Psychiatric Research 20, 31-43.

Cadoret, R. J. (1978). Evidence for the genetic inheritance of primary affective disorder in adoptees. American Journal of Psychiatry 135, 463-466.

Dickenson, A. \& Mackintosh, N. J. (1978). Classical conditioning in animals. Annual Review of Psychology 29, 587-612.
Farmer, D., Roffoli, T. \& Wolfram, S. (eds) (1984). Cellular Automata (Proceedings of an Interdisciplinary Workshop). North-Holland: Amsterdam.

Feinstein, A. R. (1974). The derangements of the 'range of normal'. Clinical Pharmacology and Therapeutics 15, 528-540. Reprinted in Feinstein, A. R. (1977). Clinical Biostatistics. Mosby: St Louis.

Fisher, R. A. (1918). The correlation between relatives on the supposition of Mendelian inheritance. Transactions of the Royal Society of Edinburgh 52, 399-433.

Fisher, R. A. (1958). The Genetic Theory of Natural Selection. Dover: New York.

Folstein, M. F., De Paulo, J. R. \& Trepp, K. (1982). Unusual mood stability in patients taking lithum. British Journal of Psychiatry 140, 188-191. 
Galen, R. S. \& Gambino, S. R. (1975). Beyond Normality. The Predictive Value and Effictency of Medical Diagnosis. Wiley: New York.

Garrod, A. E. (1909). Inborn Errors of Metabolism. Frowde, Hodder and Stoughton: London.

Gusella, J. F., Wexler, N. S., Conneally, P. M , Naylor, S. L., Anderson, M. A., Tanzi, R. E., Watkins, P. S., Ottina, K., Wallace, M. R., Sakaguchi, A. Y., Young, A. B., Shoulson, F., Bonilla, E. \& Martin, J. B. (1983). A polymorphic DNA marker genetically linked to Huntington's disease. Nature 306, 234-238.

Harker, L. A. \& Scott, C. R. (1977). Platelets in homocystinuria. New England Journal of Medicine 296, 818.

Jensen, A. R. (1969). How much can we boost IQ and scholastic achievement? Harvard Educational Review 39, 1-123.

Judd, L. L., Hubbard, B. \& Janowski, D. S. (1977). The effect of lithium carbonate on affect, mood, and personality of normal subjects. Archives of General Psychiatry 34, 346-351.

Klawans, H. L. \& Goetz, C. G. (1980). Levodopa and presymptomatic detection of Huntington's disease. Eight year follow-up. New England Journal of Medicine 302, 1090.

Levy, H. L. (1973). Genetic screening. Advances in Human Genetics 4, 1-104.

Morales, A. J., Murphy, E. A. \& Krush, A. J. (1984). The bingo model of survivorship II. Statistical aspects of the bingo model of multiplicity 1 with application to hereditary polyposis of the colon. American Journal of Medical Genetics 17, 783-801.

Murphy, E. A. (1966). A scientific viewpoint on normalcy. Perspectives in Biology and Medicine 9, 333-348.

Murphy, E. A. (1971). The estimation of blood platelet survival III. The robustness of the basic models. Thrombosis et Diathesis Haemorrhagica 26, 431-449.

Murphy, E. A. $(1981 a)$. The pursuit of the minor premise. A comment on normality. Metantedicine 2, 283-299.

Murphy, E. A. $(1981 b)$. The genetic dynamics of disease. American Journal of Medical Genetics 8, 35-52.

Murphy, E. A. \& Francis, M. E. (1971). The estimation of blood platelet survival II. The multiple hit model. Thrombosis et Diathesis Haemorrhagica 25, 53-80.
Murphy, E. A. \& Pyeritz, R. E. (1986). Homeostasis VII. A conspectus. American Journal of Medical Genetics 24, 735-751.

Murphy, E. A., Francis, M.E. \& Bollong, D. R. (1973). The estimation of blood platelet survival V. A method for the analysis of population data. Journal of Chronic Disease 26, 797-815.

Murphy, E. A., Trojak, J. E., Hou, W. \& Rohde, C. A (1981). The bingo model of survivorship. 1. Probabilistic aspects. American Journal of Medical Genetics 10, 261-277.

Opitz, J. M. (1977). The Amencan Journal of Medical Genetics Foreword. American Journal of Medical Genetics 1, 1-2.

Penrose, L. S. (1951). Measurement of pleotropic effects in phenylketonuria. Annals of Eugentcs, London 16, 134-138.

Reimann, H. A., Moadie, J, Semerdijian, S. \& Sahyoun, P. F. (1954). Periodic peritonitis, heredity and pathology. Report of seventy-two cases. Journal of American Medical Association 154, 1254-1259.

Russell, B. (1950). An Enquiry into Meaning and Truth. Allen and Unwin: London.

Seckel, H. P. G. (1960). Bird-headed Dwarfs: Studies in Developmental Anthropology Including Human Proportions. Charles C. Thomas: Springfield.

Strahan, N. V., Murphy, E. A., Fortun, N. J., Come, P. C. \& Humphries, J. O. (1983). Inheritance of mitral valve prolapse syndrome. Discussion of a three-compartmental penetrance model. American Journal of Medicine 74, 967-972.

Trojak, J. E. \& Murphy, E. A. (1983). Paradoxical fixation of deleterious alleles in two-locus systems with epistasis. American Journal of Medical Genetics 16, 493-502.

Turner, G. \& Jacobs, P. (1983). Marker X-linked mental retardation. Advances in Human Genetics 13, 83-112.

Turner, G. \& Turner, B. (1974). X-linked mental retardation. Journal of Medical Genetics 11, 109-113.

Vogel, F. \& Motulsky, G. (1979). Human Genetics; Problems and Approaches. (pp. 527-531). Springer: New York.

Walderstrom, J. \& Haeger-Aronsen, B. (1963). Different patterns of human prophyria. British Medical Journal ii, 272-276.

Webb, W. B. \& Cartwright, R. D. (1978). Sleep and dreams. Annual Review of Psychology 29, 223-252. 